\title{
Antiplane Shear Flows in Visco-plastic Solids Exhibiting Isotropic and Kinematic Hardening
}

\author{
J. M. Greenberg ${ }^{1}$ and D. R. Owen ${ }^{2}$ \\ Department of Mathematical Sciences \\ Carnegie Mellon University \\ Pittsburgh, PA 15213
}

\begin{abstract}
The authors consider antiplane shearing motions of an incompressible visco-plastic solid. The particular constitutive equation employed assumes that the stress tensor has an "elastic" component and a component which can exhibit hysteresis. The model exhibits both "kinematic" and "isotropic" hardening. Our results consist of a set of energy type estimates for the resulting system, $L_{2}$ contractivity estimates for the solution operator, and finally an analysis of the approach of our system to a "rate independent" model as a distinguished parameter describing our flow rule approaches zero. We also include some computational results for simple piecewise constant data.
\end{abstract}

${ }^{1}$ This research was partially supported by the Applied Mathematical Sciences Program, U.S. Department of Energy and the Mathematics and Computer Science Division, Army Research Office.

2 This research was partially supported by the National Science Foundation. 


\section{DISCLAIMER}

This report was prepared as an account of work sponsored by an agency of the United States Government. Neither the United States Government nor any agency thereof, nor any of their employees, makes any warranty, express or implied, or assumes any legal liability or responsibility for the accuracy, completeness, or usefulness of any information, apparatus, product, or process disclosed, or represents that its use would not infringe privately owned rights. Reference berein to any specific commercial product, process, or service by trade name, trademark, manufacturer, or otherwise does not necessarily constitute or imply its endorsement, recommendation, or favoring by the United States Government or any agency thereof. The views and opinions of authors expressed herein do not necessarily state or reflect those of the United States Government or any agency thereof. 


\section{DISCLAIMER}

Portions of this document may be illegible in electronic image products. Images are produced from the best available original document. 


\section{Introduction}

In this note we consider antiplane shear flows of an incompressible, isotropic, visco-plastic solid. This work generalizes and compliments earlier work of Greenberg [1], [2], Greenberg and Nouri [3], and Nouri and Rascle [4]. In particular, our work shows that the energy estimates previously obtained in [1] - [4] have counterparts for a broader class of visco-plastic materials.

The constitutive assumption we use postulates that the Cauchy stress tensor is additively decomposable into a component without hysteresis, i.e., that depends only on the present value of deformation gradient and an elasto-plastic component that exhibits hysteresis, i.e., that can depend on the past history of deformation gradient, and the flow rule we employ for the plastic strain tensor is basically an isotropic hardening rule with yield determined by the elasto-plastic stresses. The presence of a component without hysteresis for the stress tensor yields a model which exhibits both "kinematic" and "isotropic" hardening. Mihăilescu-Suliciu, Suliciu, and Williams [5] analyzed the relationship between solutions of ordinary differential equations that govern the motions of elasto-plastic and visco-plastic oscillators. Their constitutive assumptions also admit both kinematic and isotropic hardening.

The organization of this paper is as follows. In section 2 we develop the equations describing antiplane shear flows in visco-plastic solids. Section 3 focuses on a set of a-priori energy type estimates and also estimates which guarantee that the solution operator is a contraction in $L_{2}$. These estimates depend on a small parameter and as that parameter tends to zero they supply information about rate independent limit models. Similar estimates for a purely isotropic hardening model have been obtained by Rascle and Nouri [4]. In section 4 we present some numerical experiments.

\section{Model Development}

In antiplane shear flows of an incompressible isotropic visco-plastic solid, material points

$$
\xi=\xi_{1} \mathbf{e}_{1}+\xi_{2} \mathbf{e}_{2}+\xi_{3} \mathbf{e}_{3}
$$

move to ${ }^{1}$

$$
\mathbf{x}=x_{1} \mathbf{e}_{1}+x_{2} \mathbf{e}_{2}+x_{3} \mathbf{e}_{3}
$$

where

$$
x_{1}=\xi_{1}, x_{2}=\xi_{2}, \text { and } x_{3}=\xi_{3}+\phi\left(\xi_{1}, \xi_{2}, t_{1}\right) .
$$

For such motions the Eulerian velocity field is given by

$$
\mathbf{u}\left(x_{1}, x_{2}, x_{3}, t_{1}\right)=w\left(x_{1}, x_{2}, t_{1}\right) \mathbf{e}_{3}
$$

where

$$
w\left(x_{1}, x_{2}, t_{1}\right)=\frac{\partial \phi}{\partial t_{1}}\left(x_{1}, x_{2}, t_{1}\right)
$$

and the shear strains are given by

$$
\gamma_{1}=\frac{\partial \phi}{\partial x_{1}} \text { and } \gamma_{2}=\frac{\partial \phi}{\partial x_{2}} .
$$
${ }_{1} \mathbf{e}_{1}=\left(\begin{array}{l}1 \\ 0 \\ 0\end{array}\right), \mathbf{e}_{2}=\left(\begin{array}{l}0 \\ 1 \\ 0\end{array}\right)$, and $\mathbf{e}_{3}=\left(\begin{array}{l}0 \\ 0 \\ 1\end{array}\right)$ are the standard basis for elements for $R^{3}$ and $\mathbf{e}_{i} \otimes \mathbf{e}_{j}=\mathbf{e}_{i} \mathbf{e}_{j}^{T}$ are the
standard basis elements for linear operators from $R^{3}$ to $R^{3}$ 
The latter are related to the former by the compatibility relations

$$
\frac{\partial \gamma_{1}}{\partial t_{1}}=\frac{\partial w}{\partial x_{1}} \quad \text { and } \quad \frac{\partial \gamma_{2}}{\partial t_{1}}=\frac{\partial w}{\partial x_{2}} .
$$

The deformation gradient, $F$, associated with this motion is given by

$$
F=\left(\begin{array}{ccc}
1 & 0 & 0 \\
0 & 1 & 0 \\
\gamma_{1} & \gamma_{2} & 1
\end{array}\right)
$$

Our basic constitutive assumption postulates a plastic deformation tensor, $P$, of the same form as $F$; that is

$$
P=\left(\begin{array}{ccc}
1 & 0 & 0 \\
0 & 1 & 0 \\
p_{1} & p_{2} & 1
\end{array}\right)
$$

where $p_{1}$ and $p_{2}$ are functions of $x_{1}, x_{2}$, and $t_{1}$, and postulates that the Cauchy Stress tensor take the form

$$
T=-\pi I+\mathcal{S}_{1}+\mathcal{S}_{2}
$$

Here $\pi$ is the hydrostatic pressure,

$$
\mathcal{S}_{1}=\beta\left(F F^{T}-\frac{1}{3} \operatorname{trace}\left(F F^{T}\right) I\right)
$$

or equivalently

$$
\begin{gathered}
\mathcal{S}_{1}=\beta\left(\begin{array}{ccc}
-\frac{1}{3}\left(\gamma_{1}^{2}+\gamma_{2}^{2}\right) & 0 & \gamma_{1} \\
0 & -\frac{1}{3}\left(\gamma_{1}^{2}+\gamma_{2}^{2}\right) & \gamma_{2} \\
\gamma_{1} & \gamma_{2} & \frac{2}{3}\left(\gamma_{1}^{2}+\gamma_{2}^{2}\right)
\end{array}\right), \\
\mathcal{S}_{2}=\mu\left(R R^{T}-\frac{1}{3} \operatorname{trace}\left(R R^{T}\right) I\right)
\end{gathered}
$$

or equivalently

$$
\begin{gathered}
\mathcal{S}_{2}=\mu\left(\begin{array}{ccc}
-\frac{1}{3}\left(r_{1}^{2}+r_{2}^{2}\right) & 0 & r_{1} \\
0 & -\frac{1}{3}\left(r_{1}^{2}+r_{2}^{2}\right) & r_{2} \\
r_{1} & r_{2} & \frac{2}{3}\left(r_{1}^{2}+r_{2}^{2}\right)
\end{array}\right), \\
R=F P^{-1}=P^{-1} F
\end{gathered}
$$

or equivalently

$$
R=\left(\begin{array}{ccc}
1 & 0 & 0 \\
0 & 1 & 0 \\
r_{1} & r_{2} & 1
\end{array}\right)=\left(\begin{array}{ccc}
1 & 0 & 0 \\
0 & 1 & 0 \\
\gamma_{1}-p_{1} & \gamma_{2}-p_{2} & 1
\end{array}\right)
$$

and $\beta$ and $\mu$ are positive constants. Equations (2.9) - (2.14) imply that $\mathcal{S}_{1}$ is an isotropic trace free function of $F$ that has the interpretation of a stress without hysteresis, whereas $\mathcal{S}_{2}$ is an isotropic trace free function of $R$ that is interpreted as the "elasto-plastic" stress and exhibits hysteresis for appropriate deformation histories. 
In what follows it will be convenient to let

$$
s_{1}:=\mu\left(\gamma_{1}-p_{1}\right) \quad \text { and } \quad s_{2}:=\mu\left(\gamma_{2}-p_{2}\right)
$$

and to use $s_{1}\left(x_{1}, x_{2}, t_{1}\right), s_{2}\left(x_{1}, x_{2}, t_{1}\right), p_{1}\left(x_{1}, x_{2}, t_{1}\right)$ and $p_{2}\left(x_{1}, x_{2}, t_{1}\right)$ as the basic descriptors of our system. With this choice we have

and

$$
\begin{gathered}
F=\left(\begin{array}{ccc}
1 & 0 & 0 \\
0 & 1 & 0 \\
\frac{s_{1}}{\mu}+p_{1} & \frac{s_{2}}{\mu}+p_{2} & 1
\end{array}\right), \\
R=\left(\begin{array}{ccc}
1 & 0 & 0 \\
0 & 1 & 0 \\
\frac{s_{1}}{\mu} & \frac{s_{2}}{\mu} & 1
\end{array}\right), \\
\mathcal{S}_{2}=\left(\begin{array}{ccc}
-\frac{1}{3 \mu}\left(s_{1}^{2}+s_{2}^{2}\right) & 0 & s_{1} \\
0 & -\frac{1}{3 \mu}\left(s_{1}^{2}+s_{2}^{2}\right) & s_{2} \\
s_{1} & & s_{2}
\end{array}\right),
\end{gathered}
$$

$\mathcal{S}_{1}=\beta\left(\begin{array}{ccc}-\frac{1}{3}\left(\left(\frac{s_{1}}{\mu}+p_{1}\right)^{2}+\left(\frac{s_{2}}{\mu}+p_{2}\right)^{2}\right) & 0 & \frac{s_{1}}{\mu}+p_{1} \\ 0 & -\frac{1}{3}\left(\left(\frac{s_{1}}{\mu}+p_{1}\right)^{2}+\left(\frac{s_{2}}{\mu}+p_{2}\right)^{2}\right) & \frac{s_{2}}{\mu}+p_{2} \\ \frac{s_{1}}{\mu}+p_{1} & \frac{s_{2}}{\mu}+p_{2} & \frac{2}{3}\left(\left(\frac{s_{1}}{\mu}+p_{1}\right)^{2}+\left(\frac{s_{2}}{\mu}+p_{2}\right)^{2}\right)\end{array}\right)$.

Balance of momentum in the $\mathbf{e}_{1}$ and $\mathbf{e}_{2}$ directions implies that

$$
\begin{aligned}
& \frac{\partial}{\partial x_{1}}\left(\pi+\frac{\left(s_{1}^{2}+s_{2}^{2}\right)}{3 \mu}+\frac{\beta}{3}\left(\left(\frac{s_{1}}{\mu}+p_{1}\right)^{2}+\left(\frac{s_{2}}{\mu}+p_{2}\right)^{2}\right)\right)=0, \\
& \frac{\partial}{\partial x_{2}}\left(\pi+\frac{\left(s_{1}^{2}+s_{2}^{2}\right)}{3 \mu}+\frac{\beta}{3}\left(\left(\frac{s_{1}}{\mu}+p_{1}\right)^{2}+\left(\frac{s_{2}}{\mu}+p_{2}\right)^{2}\right)\right)=0,
\end{aligned}
$$

and thus that the pressure, $\pi$, is given by

$$
\pi=\pi_{0}\left(x_{3}, t\right)-\frac{\left(s_{1}^{2}+s_{2}^{2}\right)}{3 \mu}-\frac{\beta}{3}\left(\left(\frac{s_{1}}{\mu}+p_{1}\right)^{2}+\left(\frac{s_{2}}{\mu}+p_{2}\right)^{2}\right) .
$$

Balance of momentum in the $\mathbf{e}_{\mathbf{3}}$ direction yields

$$
\rho_{0} \frac{\partial w}{\partial t_{1}}-\frac{\partial}{\partial x_{1}}\left(s_{1}+\beta\left(\frac{s_{1}}{\mu}+p_{1}\right)\right)-\frac{\partial}{\partial x_{2}}\left(s_{2}+\beta\left(\frac{s_{2}}{\mu}+p_{2}\right)\right)=-\frac{\partial \pi_{0}}{\partial x_{3}} .
$$


Here, $\rho_{0}$ is the constant mass density of the material. Since $\frac{\partial \pi_{0}}{\partial x_{3}}$ depends on $x_{3}$ and $t_{1}$, whereas all quantities in the left-hand side of (2.24) depend on $x_{1}, x_{2}$ and $t_{1}$, we conclude that for antiplane shear flows $\frac{\partial \pi_{0}}{\partial x_{3}}$ is independent of $x_{3}$. In what follows we shall assume this term is zero.

We now turn our attention to the flow rule for the nonconstant components $p_{1}$ and $p_{2}$ of $P$. We assume the isotropic hardening rule

$$
\frac{\partial p_{1}}{\partial t_{1}}=\frac{s_{1}}{\sqrt{s_{1}^{2}+s_{2}^{2}}} \frac{\left(\sqrt{s_{1}^{2}+s_{2}^{2}}-s_{y}(d)\right)_{+}}{\mu T_{0} \epsilon}
$$

and

$$
\frac{\partial p_{2}}{\partial t_{1}}=\frac{s_{2}}{\sqrt{s_{1}^{2}+s_{2}^{2}}} \frac{\left(\sqrt{s_{1}^{2}+s_{2}^{2}}-s_{y}(d)\right)_{+}}{\mu T_{0} \epsilon} .
$$

Here,

$$
(x)_{+}=\left\{\begin{array}{l}
0, x<0 \\
x, \quad x \geq 0
\end{array},\right.
$$

$d$ is the accumulated plastic strain and satisfies

$$
\frac{\partial d}{\partial t_{1}}=\sqrt{\left(\frac{\partial p_{1}}{\partial t_{1}}\right)^{2}+\left(\frac{\partial p_{2}}{\partial t_{1}}\right)^{2}}=\frac{\left(\sqrt{s_{1}^{2}+s_{2}^{2}}-s_{y}(d)\right)_{+}}{\mu T_{0} \epsilon}
$$

and finally $d \rightarrow s_{y}(d)$ is the yield stress which we assume satisfies

$$
0<s_{y}(0) \leq s_{y}(d), \quad 0<m \leq s_{y}^{\prime}(d) \quad \text { and } \quad-M \leq s_{y}^{\prime \prime}(d) \leq 0
$$

for $d \geq 0$. The parameter $T_{0}$ has the interpretation of a relaxation time, $\mu$ is the "shear" modulus, and $\epsilon>0$ is a dimensionless small parameter.

Equations (2.5), (2.15), and (2.24) -(2.28) combine to yield the following system for $s_{1}, s_{2}, p_{1}, p_{2}, d$, and $w$ :

$$
\begin{aligned}
& \frac{1}{\mu} \frac{\partial s_{1}}{\partial t_{1}}-\frac{\partial w}{\partial x_{1}}=-\frac{\partial p_{1}}{\partial t_{1}} \\
& \frac{1}{\mu} \frac{\partial s_{2}}{\partial t_{1}}-\frac{\partial w}{\partial x_{2}}=-\frac{\partial p_{2}}{\partial t_{1}}
\end{aligned}
$$$$
\rho_{0} \frac{\partial w}{\partial t_{1}}-\frac{\partial}{\partial x_{1}}\left(\frac{(\beta+\mu)}{\mu} s_{1}+\beta p_{1}\right)-\frac{\partial}{\partial x_{2}}\left(\frac{(\beta+\mu)}{\mu} s_{2}+\beta p_{2}\right)=0
$$$$
\frac{\partial p_{1}}{\partial t_{1}}=\frac{s_{1}}{\sqrt{s_{1}^{2}+s_{2}^{2}}} \frac{\left(\sqrt{s_{1}^{2}+s_{2}^{2}}-s_{y}(d)\right)_{+}}{\mu T_{0} \epsilon}
$$$$
\frac{\partial p_{2}}{\partial t_{1}}=\frac{s_{2}}{\sqrt{s_{1}^{2}+s_{2}^{2}}} \frac{\left(\sqrt{s_{1}^{2}+s_{2}^{2}}-s_{y}(d)\right)_{+}}{\mu T_{0} \epsilon}
$$

and

$$
\frac{\partial d}{\partial t_{1}}=\frac{\left(\sqrt{s_{1}^{2}+s_{2}^{2}}-s_{y}(d)\right)_{+}}{\mu T_{0} \epsilon} .
$$


For convenience, we put (2.30) - (2.35) into dimensionless form. We let

$$
\left\{\begin{array}{c}
x=\sqrt{\frac{\rho_{0}}{\mu+\beta}} \frac{x_{1}}{T_{0}}, \quad y=\sqrt{\frac{\rho_{0}}{\mu+\beta}} \frac{y_{1}}{T_{0}}, \quad t=\frac{t_{1}}{T_{0}} \\
u=\sqrt{\frac{\rho_{0}}{\mu+\eta}} w, \quad \tau_{1}=\frac{s_{1}}{\mu}, \quad \tau_{2}=\frac{s_{2}}{\mu} \quad \text { and } \quad \tau_{y}(d)=\frac{s_{y}(d)}{\mu} .
\end{array}\right.
$$

Then, (2.30) - (2.35) transforms to

$$
\frac{\partial \tau_{1}}{\partial t}-\frac{\partial u}{\partial x}=-\frac{\partial p_{1}}{\partial t}
$$

$$
\frac{\partial \tau_{2}}{\partial t}-\frac{\partial u}{\partial y}=-\frac{\partial p_{2}}{\partial t}
$$

$$
\frac{\partial u}{\partial t}-\frac{\partial}{\partial x}\left(\tau_{1}+\frac{\beta}{\mu+\beta} p_{1}\right)-\frac{\partial}{\partial y}\left(\tau_{2}+\frac{\beta}{\mu+\beta} p_{2}\right)=0
$$

$$
\frac{\partial p_{1}}{\partial t}=\frac{\tau_{1}\left(\sqrt{\tau_{1}^{2}+\tau_{2}^{2}}-\tau_{y}(d)\right)_{+}}{\epsilon \sqrt{\tau_{1}^{2}+\tau_{2}^{2}}}
$$

$$
\frac{\partial p_{2}}{\partial t}=\frac{\tau_{2}\left(\sqrt{\tau_{1}^{2}+\tau_{2}^{2}}-\tau_{y}(d)\right)_{+}}{\epsilon \sqrt{\tau_{1}^{2}+\tau_{2}^{2}}}
$$

and

$$
\frac{\partial d}{\partial t}=\frac{\left(\sqrt{\tau_{1}^{2}+\tau_{2}^{2}}-\tau_{y}(d)\right)_{+}}{\epsilon} \geq 0 .
$$

In section 3 we shall present a set of energy type estimates for (2.37) - (2.42).

\section{$3 \quad$ Energy Estimates}

In this section we shall focus on a series of energy type estimates for solutions of the Cauchy problem for (2.37) - (2.42). We shall continually use an integral identity, presented in (3.2) below, for solutions of the divergence identity:

$$
\frac{\partial f}{\partial t}-\frac{\partial q_{1}}{\partial x}-\frac{\partial q_{2}}{\partial y}=g
$$

The basic identities are 


$$
\begin{aligned}
& \frac{d}{d s} \underset{\left\{\sqrt{\left(x-x_{0}\right)^{2}+\left(y-y_{0}\right)^{2}} \leq R+t-s\right\}}{ } f(x, y, s) d x d y= \\
& (R+t-s) \int_{0}^{2 \pi}\left[q_{1} \cos \theta+q_{2} \sin \theta-f\right]\left(x_{0}+(R+t-s) \cos \theta, y_{0}+(R+t-s) \sin \theta, s\right) d \theta \\
& +\quad \iint(x, y, s) d x d y \\
& \left\{\sqrt{\left(x-x_{0}\right)^{2}+\left(y-y_{0}\right)^{2}} \leq R+t-s\right\}
\end{aligned}
$$

and

$$
\iint_{\left\{\sqrt{\left(x-x_{0}\right)^{2}+\left(y-y_{0}\right)^{2}} \leq R\right\}} f(x, y, s) d x d y=
$$

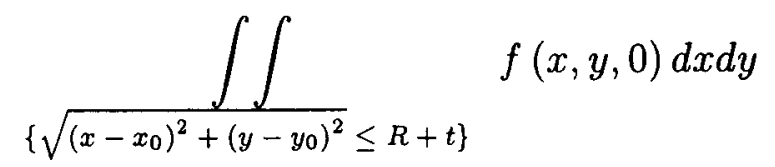$$
+\int_{0}^{t}(R+t-s)\left(\int_{0}^{2 \pi}\left[q_{1} \cos \theta+q_{2} \sin \theta-f\right]\left(x_{0}+(R+t+s) \cos \theta, y_{0}+(R+t-s) \sin \theta, s\right) d \theta\right) d s
$$

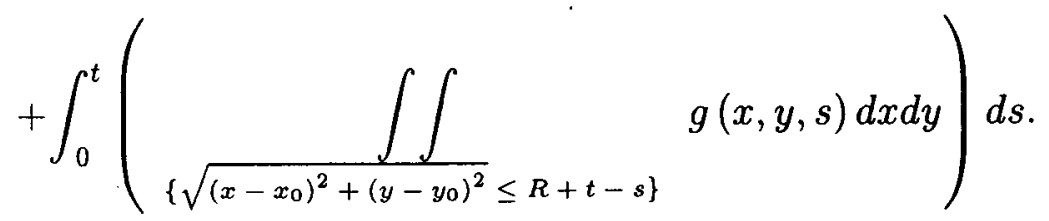

Our first identity is obtained by multiplying (2.37) by $\tau_{1},(2.38)$ by $\tau_{2},(2.39)$ by $u$ and $(2.42)$ by $\frac{\mu}{\beta+\mu} \tau_{y}(d)$ and adding the equations. The result is that (3.1) holds with

$$
\begin{gathered}
f=\frac{\mu\left(\tau_{1}^{2}+\tau_{2}^{2}\right)}{2(\mu+\beta)}+\frac{\beta\left(\left(\tau_{1}+p_{1}\right)^{2}+\left(\tau_{2}+p_{2}\right)^{2}\right)}{2(\mu+\beta)}+\frac{u^{2}}{2}+\frac{\mu}{\mu+\beta} \int_{0}^{d} \tau_{y}(\eta) d \eta \\
q_{1}=u\left(\frac{\mu}{\mu+\beta} \tau_{1}+\frac{\beta}{\mu+\beta}\left(\tau_{1}+p_{1}\right)\right) \\
q_{2}=u\left(\frac{\mu}{\mu+\beta} \tau_{2}+\frac{\beta}{\mu+\beta}\left(\tau_{2}+p_{2}\right)\right)
\end{gathered}
$$

and

$$
g=\frac{-\mu}{\epsilon(\mu+\beta)}\left(\left(\sqrt{\tau_{1}^{2}+\tau_{2}^{2}}-\tau_{y}(d)\right)_{+}\right)^{2}
$$


The observation that

$$
q_{1} \cos \theta+q_{2} \sin \theta \leq|u|\left(\frac{\mu}{\mu+\beta} \sqrt{\tau_{1}^{2}+\tau_{2}^{2}}+\frac{\beta}{\mu+\beta} \sqrt{\left(\tau_{1}+p_{1}\right)^{2}+\left(\tau_{2}+p_{2}\right)^{2}}\right)
$$

implies that

$$
\begin{aligned}
& q_{1} \cos \theta+q_{2} \sin \theta-f \leq \\
& -\frac{\mu}{2(\mu+\beta)}\left(|u|-\sqrt{\tau_{1}^{2}+\tau_{2}^{2}}\right)^{2}-\frac{\beta}{2(\mu+\beta)}\left(|u|-\sqrt{\left(\tau_{1}+p_{1}\right)^{2}+\left(\tau_{2}+p_{2}\right)^{2}}\right)^{2} \\
& -\frac{\mu}{\mu+\beta} \int_{0}^{d} \tau_{y}(\eta) d \eta \leq 0
\end{aligned}
$$

and thus the last two integrals on the right-hand side of (3.2) are less than or equal to zero. ${ }^{2}$

To obtain the contractivity of the solution operator for $(2.37)-(2.42)$ we look at two solutions to (2.37) - (2.42) which we label $a$ and $b$, respectively. Their differences satisfy

$$
\begin{gathered}
\frac{\partial}{\partial t}\left(\tau_{1}^{a}-\tau_{1}^{b}\right)-\frac{\partial}{\partial x}\left(u^{a}-u^{b}\right)=-\frac{\partial}{\partial t}\left(p_{1}^{a}-p_{1}^{b}\right) \\
\frac{\partial}{\partial t}\left(\tau_{2}^{a}-\tau_{2}^{b}\right)-\frac{\partial}{\partial y}\left(u^{a}-u^{b}\right)=-\frac{\partial}{\partial t}\left(p_{2}^{a}-p_{2}^{b}\right) \\
\frac{\partial}{\partial t}\left(u^{a}-u^{b}\right)-\frac{\partial}{\partial x}\left(\tau_{1}^{a}-\tau_{1}^{b}+\frac{\beta\left(p_{1}^{a}-p_{1}^{b}\right)}{\mu+\beta}\right)-\frac{\partial}{\partial y}\left(\tau_{2}^{a}-\tau_{2}^{b}+\frac{\beta\left(p_{2}^{a}-p_{2}^{b}\right)}{\mu+\beta}\right)=0 \\
\frac{\partial}{\partial t}\left(p_{1}^{a}-p_{1}^{b}\right)=\frac{\tau_{1}^{a}\left(\sqrt{\left(\tau_{1}^{a}\right)^{2}+\left(\tau_{2}^{a}\right)^{2}}-\tau_{y}\left(d^{a}\right)\right)_{+}}{\epsilon \sqrt{\left(\tau_{1}^{a}\right)^{2}+\left(\tau_{2}^{a}\right)^{2}}}-\frac{\tau_{1}^{b}\left(\sqrt{\left(\tau_{1}^{b}\right)^{2}+\left(\tau_{2}^{b}\right)^{2}}-\tau_{y}\left(d^{b}\right)\right)_{+}}{\epsilon \sqrt{\left(\tau_{1}^{b}\right)^{2}+\left(\tau_{2}^{b}\right)^{2}}} \\
\frac{\partial}{\partial t}\left(p_{2}^{a}-p_{2}^{b}\right)=\frac{\tau_{2}^{a}\left(\sqrt{\left(\tau_{1}^{a}\right)^{2}+\left(\tau_{2}^{a}\right)^{2}}-\tau_{y}\left(d^{a}\right)\right)_{+}}{\epsilon \sqrt{\left(\tau_{1}^{a}\right)^{2}+\left(\tau_{2}^{a}\right)^{2}}}-\frac{\tau_{2}^{b}\left(\sqrt{\left(\tau_{1}^{b}\right)^{2}+\left(\tau_{2}^{b}\right)^{2}}-\tau_{y}\left(d^{b}\right)\right)_{+}}{\epsilon \sqrt{\left(\tau_{1}^{b}\right)^{2}+\left(\tau_{2}^{b}\right)^{2}}}
\end{gathered}
$$

and

$$
\frac{\partial}{\partial t}\left(d^{a}-d^{b}\right)=\frac{\left(\sqrt{\left(\tau_{1}^{a}\right)^{2}+\left(\tau_{2}^{a}\right)^{2}}-\tau_{y}\left(d^{a}\right)\right)_{+}}{\epsilon}-\frac{\left(\sqrt{\left(\tau_{1}^{b}\right)^{2}+\left(\tau_{2}^{b}\right)^{2}}-\tau_{y}\left(d^{b}\right)\right)_{+}}{\epsilon}
$$

We next multiply (3.9) by $\left(\tau_{1}^{a}-\tau_{1}^{b}\right),(3.10)$ by $\left(\tau_{2}^{a}-\tau_{2}^{b}\right),(3.11)$ by $\left(u^{a}-u^{b}\right)$, and (3.14) by

$$
\frac{\mu}{\mu+\beta}\left(\tau_{y}\left(d^{a}\right)-\tau_{y}\left(d^{b}\right)\right)=\frac{\mu}{\mu+\beta}\left(\int_{0}^{1} \tau_{y}^{\prime}\left((1-\eta) d^{b}+\eta d^{a}\right) d \eta\right)\left(d^{a}-d^{b}\right)
$$

${ }^{2}$ We are, of course, using the fact that $d \geq 0, d_{t} \geq 0$, and that $0 \leq \tau_{y}(0) d \leq \int_{0}^{d} \tau_{y}(\eta) d y$ 
and add the resulting equations. The result is that (3.1) holds where now

$$
f=\frac{1}{2}\left(\frac{\mu\left(\left(\tau_{1}^{a}-\tau_{1}^{b}\right)^{2}+\left(\tau_{2}^{a}-\tau_{2}^{b}\right)^{2}\right)}{\mu+\beta}+\frac{\beta\left(\left(\tau_{1}^{a}-\tau_{1}^{b}+p_{1}^{a}-p_{1}^{b}\right)^{2}+\left(\tau_{2}^{a}-\tau_{2}^{b}+p_{2}^{a}-p_{2}^{b}\right)^{2}\right)}{\mu+\beta}\right.
$$

$$
\left.+\left(u^{a}-u^{b}\right)^{2}+\frac{\mu}{\mu+\beta}\left(\int_{0}^{1} \tau_{y}^{\prime}\left((1-\eta) d^{b}+\eta d^{a}\right) d \eta\right)\left(d^{a}-d^{b}\right)^{2}\right)
$$

$$
\begin{aligned}
& q_{1}=\left(u^{a}-u^{b}\right)\left(\frac{\mu}{\mu+\beta}\left(\tau_{1}^{a}-\tau_{1}^{b}\right)+\frac{\beta}{\mu+\beta}\left(\tau_{1}^{a}-\tau_{1}^{b}+p_{2}^{a}-p_{2}^{b}\right)\right), \\
& q_{2}=\left(u^{a}-u^{b}\right)\left(\frac{\mu}{\mu+\beta}\left(\tau_{2}^{a}-\tau_{2}^{b}\right)+\frac{\beta}{\mu+\beta}\left(\tau_{2}^{a}-\tau_{2}^{b}+p_{2}^{a}-p_{2}^{b}\right)\right),
\end{aligned}
$$

and

$$
g=\frac{\mu}{\mu+\beta}\left(g_{1}+\frac{g_{2}}{\epsilon}\right)
$$

where

$$
g_{1}=\frac{1}{2}\left(\int_{0}^{1} r_{y}^{\prime \prime}\left((1-\eta) d^{b}+\eta d^{a}\right)\left((1-\eta) d_{t}^{b}+\eta d_{t}^{a}\right) d \eta\right)\left(d^{a}-d^{b}\right)^{2}
$$

and

$$
\begin{array}{r}
g_{2}=\left(\tau_{y}\left(d^{a}\right)-\tau_{y}\left(d^{b}\right)\right)\left(\left(\sqrt{\left(\tau_{1}^{a}\right)^{2}+\left(\tau_{2}^{a}\right)^{2}}-\tau_{y}\left(d^{a}\right)\right)_{+}-\left(\sqrt{\left(\tau_{1}^{b}\right)^{2}+\left(\tau_{2}^{b}\right)^{2}}-\tau_{y}\left(d^{b}\right)\right)_{+}\right) \\
\left.-\left(\tau_{1}^{a}-\tau_{1}^{b}\right)\left(\frac{\tau_{1}^{a}\left(\sqrt{\left(\tau_{1}^{a}\right)^{2}+\left(\tau_{2}^{a}\right)^{2}}-\tau_{y}\left(d^{a}\right)\right)_{+}}{\sqrt{\left(\tau_{1}^{a}\right)^{2}+\left(\tau_{2}^{a}\right)^{2}}}\right) \frac{\tau_{1}^{b}\left(\sqrt{\left(\tau_{1}^{b}\right)^{2}+\left(\tau_{2}^{b}\right)^{2}}-\tau_{y}\left(d^{b}\right)\right)_{+}}{\sqrt{\left(\tau_{1}^{b}\right)^{2}+\left(\tau_{2}^{b}\right)^{2}}}\right) \\
-\left(\tau_{2}^{a}-\tau_{2}^{b}\right)\left(\frac{\tau_{2}^{a}\left(\sqrt{\left(\tau_{1}^{a}\right)^{2}+\left(\tau_{2}^{a}\right)^{2}}-\tau_{y}\left(d^{a}\right)\right)_{+}}{\sqrt{\left(\tau_{1}^{a}\right)^{2}+\left(\tau_{2}^{a}\right)^{2}}}-\frac{\tau_{2}^{b}\left(\sqrt{\left(\tau_{1}^{b}\right)^{2}+\left(\tau_{2}^{b}\right)^{2}}-\tau_{y}\left(d^{b}\right)\right)_{+}}{\sqrt{\left(\tau_{1}^{b}\right)^{2}+\left(\tau_{2}^{b}\right)^{2}}}\right)
\end{array}
$$

The arguments leading to (3.8) yield the inequality

$$
\begin{aligned}
& q_{1} \cos \theta+q_{2} \sin \theta-f \leq-\frac{\mu}{2(\mu+\beta)}\left(\left|u^{a}-u^{b}\right|-\sqrt{\left(\tau_{1}^{a}-\tau_{1}^{b}\right)^{2}+\left(\tau_{2}^{a}-\tau_{2}^{b}\right)^{2}}\right)^{2} \\
& -\frac{\beta}{2(\mu+\beta)}\left(\left|u^{a}-u^{b}\right|-\sqrt{\left(\tau_{1}^{a}-\tau_{1}^{b}+p_{1}^{a}-p_{1}^{b}\right)^{2}+\left(\tau_{2}^{a}-\tau_{2}^{b}+p_{2}^{a}-p_{2}^{b}\right)^{2}}\right)^{2} \\
& -\frac{\mu}{2(\mu+\beta)}\left(\int_{0}^{1} \tau_{y}^{\prime}\left((1-\eta) d^{b}+\eta d^{a}\right) d \eta\right)\left(d^{a}-d^{b}\right)^{2} \leq 0 .
\end{aligned}
$$


We further note that the hypotheses that $0<\tau_{y}(0) \leq \tau_{y}(d), 0<\frac{m}{\mu} \leq \tau_{y}^{\prime}(d)$, and $-\frac{M}{\mu} \leq \tau_{y}^{\prime \prime}(d) \leq 0$ for $d \geq 0$ guarantees that $g_{1} \leq 0$. Thus, to show that

$$
\frac{\iint{ }_{\left\{\sqrt{\left(x-x_{0}\right)^{2}+\left(y-y_{0}\right)^{2}} \leq R\right\}}}{f(x, y, t) d x d y \leq} \underset{\left\{\sqrt{\left(x-x_{0}\right)^{2}+\left(y-y_{0}\right)^{2}} \leq R+t\right\}}{\iint} f(x, y, 0) d x d y
$$

where $f$ is given by (3.15) it suffices to show that $g_{2} \leq 0$. We first note that $g_{2}$ may be written as

$$
\begin{aligned}
g_{2}= & -\left(\sqrt{\left(\tau_{1}^{a}\right)^{2}+\left(\tau_{2}^{a}\right)^{2}}-\tau_{y}\left(d^{a}\right)\right)_{+}\left(\sqrt{\left(\tau_{1}^{a}\right)^{2}+\left(\tau_{2}^{a}\right)^{2}}-\tau_{y}\left(d^{a}\right)\right) \\
& -\left(\sqrt{\left(\tau_{1}^{b}\right)^{2}+\left(\tau_{2}^{b}\right)^{2}}-\tau_{y}\left(d^{b}\right)\right)_{+}\left(\sqrt{\left(\tau_{1}^{b}\right)^{2}+\left(\tau_{2}^{b}\right)^{2}}-\tau_{y}\left(d^{b}\right)\right) \\
& +\left(\sqrt{\left(\tau_{1}^{a}\right)^{2}+\left(\tau_{2}^{a}\right)^{2}}-\tau_{y}\left(d^{a}\right)\right)_{+}\left(\frac{\tau_{1}^{a} \tau_{1}^{b}+\tau_{2}^{a} \tau_{2}^{b}}{\sqrt{\left(\tau_{1}^{a}\right)^{2}+\left(\tau_{2}^{a}\right)^{2}}}-\tau_{y}\left(d^{b}\right)\right) \\
& +\left(\sqrt{\left(\tau_{1}^{b}\right)^{2}+\left(\tau_{2}^{b}\right)^{2}}-\tau_{y}\left(d^{b}\right)\right)_{+}\left(\frac{\tau_{1}^{a} \tau_{1}^{b}+\tau_{2}^{a} \tau_{2}^{b}}{\sqrt{\left(\tau_{1}^{b}\right)^{2}+\left(\tau_{2}^{b}\right)^{2}}}-\tau_{y}\left(d^{a}\right)\right)
\end{aligned}
$$

and (3.23), together with

$$
\tau_{1}^{a} \tau_{1}^{b}+\tau_{2}^{a} \tau_{2}^{b} \leq \sqrt{\left(\tau_{1}^{a}\right)^{2}+\left(\tau_{1}^{b}\right)^{2}} \sqrt{\left(\tau_{2}^{a}\right)^{2}+\left(\tau_{2}^{b}\right)^{2}}
$$

implies that

$$
\begin{aligned}
g_{2} & \leq-\left(\sqrt{\left(\tau_{1}^{a}\right)^{2}-\left(\tau_{2}^{a}\right)^{2}}-\tau_{y}\left(d^{a}\right)\right)_{+}\left(\sqrt{\left(\tau_{1}^{a}\right)^{2}+\left(\tau_{2}^{a}\right)^{2}}-\tau_{y}\left(d^{a}\right)\right) \\
& -\left(\sqrt{\left(\tau_{1}^{b}\right)^{2}+\left(\tau_{2}^{b}\right)^{2}}-\tau_{y}\left(d^{b}\right)\right)_{+}\left(\sqrt{\left(\tau_{1}^{b}\right)^{2}+\left(\tau_{2}^{b}\right)^{2}}-\tau_{y}\left(d^{b}\right)\right) \\
& +\left(\sqrt{\left(\tau_{1}^{a}\right)^{2}+\left(\tau_{2}^{a}\right)^{2}}-\tau_{y}\left(d^{a}\right)\right)_{+}\left(\sqrt{\left(\tau_{1}^{b}\right)^{2}+\left(\tau_{2}^{b}\right)^{2}}-\tau_{y}\left(d^{b}\right)\right) \\
& +\left(\sqrt{\left(\tau_{1}^{b}\right)^{2}+\left(\tau_{2}^{b}\right)^{2}}-\tau_{y}\left(d^{b}\right)\right)_{+}\left(\sqrt{\left(\tau_{1}^{a}\right)^{2}+\left(\tau_{2}^{a}\right)^{2}}-\tau_{y}\left(d^{a}\right)\right)
\end{aligned}
$$


or that

$$
\begin{gathered}
g_{2}=-\left(\left(\sqrt{\left(\tau_{1}^{a}\right)^{2}+\left(\tau_{2}^{a}\right)^{2}}-\tau_{y}\left(d^{a}\right)\right)_{+}-\left(\sqrt{\left(\tau_{1}^{b}\right)^{2}+\left(\tau_{2}^{b}\right)^{2}}-\tau_{y}\left(d^{b}\right)\right)_{+}\right)^{2} \\
(3.26)+\left(\sqrt{\left(\tau_{1}^{a}\right)^{2}+\left(\tau_{2}^{a}\right)^{2}}-\tau_{y}\left(d^{a}\right)\right)_{+}\left(\sqrt{\left(\tau_{1}^{b}\right)^{2}+\left(\tau_{2}^{b}\right)^{2}}-\tau_{y}\left(d^{b}\right)-\left(\sqrt{\left(\tau_{1}^{b}\right)^{2}+\left(\tau_{2}^{b}\right)^{2}}-\tau_{y}\left(d^{b}\right)\right)_{+}\right) \\
+\left(\sqrt{\left(\tau_{1}^{b}\right)^{2}+\left(\tau_{2}^{b}\right)^{2}}-\tau_{y}\left(d^{b}\right)\right)_{+}\left(\sqrt{\left(\tau_{1}^{a}\right)^{2}+\left(\tau_{2}^{a}\right)^{2}}-\tau_{y}\left(d^{a}\right)-\left(\sqrt{\left(\tau_{1}^{a}\right)^{2}+\left(\tau_{2}^{a}\right)^{2}}-\tau_{y}\left(d^{a}\right)\right)_{+}\right) .
\end{gathered}
$$

Since each of the terms on the right-hand side of (3.26) is nonpositive we conclude that $g_{2} \leq 0$ and thus (3.22) holds, which is the desired contractivity of the solution operator associated with (2.37) - (2.42). Similar arguments have been used previously by Greenberg and Nouri [3] and Nouri and Rascle [4].

Our next objective is a set of derivative estimates for solutions of the Cauchy problem for (2.37) - (2.42). In what follows $D$ will be one of the following operators: $\frac{\partial}{\partial t}$, $\frac{\partial}{\partial x}$, or $\frac{\partial}{\partial y}$. Differentiation of the original system yields the following equations for $D \tau_{1}, D \tau_{2}, D u, D p_{1}, D p_{2}$, and $D d$ :

$$
\begin{gathered}
\frac{\partial}{\partial t}\left(D \tau_{1}\right)-\frac{\partial}{\partial x}(D u)=-\frac{\partial}{\partial t}\left(D p_{1}\right), \\
\frac{\partial}{\partial t}\left(D \tau_{2}\right)-\frac{\partial}{\partial y}(D u)=-\frac{\partial}{\partial t}\left(D p_{2}\right), \\
\frac{\partial}{\partial t}(D u)-\frac{\partial}{\partial x}\left(D \tau_{1}+\frac{\beta}{\mu+\beta} D p_{1}\right)-\frac{\partial}{\partial y}\left(D \tau_{2}+\frac{\beta}{\mu+\beta} D p_{2}\right)=0, \\
\frac{\partial}{\partial t}\left(D p_{1}\right)=\frac{\tau_{1}}{\epsilon \sqrt{\tau_{1}^{2}+\tau_{2}^{2}}} H\left(\sqrt{\tau_{1}^{2}+\tau_{2}^{2}}-\tau_{y}(d)\right)\left(\frac{\tau_{1} D \tau_{1}+\tau_{2} D \tau_{2}}{\sqrt{\tau_{1}^{2}+\tau_{2}^{2}}}-\tau_{y}^{\prime}(d) D d\right) \\
+\frac{\left(\sqrt{\tau_{1}^{2}+\tau_{2}^{2}}-\tau_{y}(d)\right)_{+}}{\left.\epsilon\left(\tau_{1}^{2}+\tau_{2}^{2}\right)^{3 / 2} D \tau_{1}-\tau_{1} \tau_{2} D \tau_{2}\right),} \\
\frac{\partial}{\partial t}\left(D p_{2}\right)=\frac{\tau_{2}}{\epsilon \sqrt{\tau_{1}^{2}+\tau_{2}^{2}}} H\left(\sqrt{\tau_{1}^{2}+\tau_{2}^{2}}-\tau_{y}(d)\right)\left(\frac{\tau_{1} D \tau_{1}+\tau_{2} D \tau_{2}}{\sqrt{\tau_{1}^{2}+\tau_{2}^{2}}}-\tau_{y}^{\prime}(d) D d\right) \\
+\frac{\left(\sqrt{\tau_{1}^{2}+\tau_{2}^{2}}-\tau_{y}(d)\right)_{+}}{\left.\epsilon\left(\tau_{1}^{2}+\tau_{2}^{2}\right)^{3 / 2} D \tau_{2}-\tau_{1} \tau_{2} D \tau_{1}\right),}
\end{gathered}
$$

and

$$
\frac{\partial}{\partial t}(D d)=\frac{H\left(\sqrt{\tau_{1}^{2}+\tau_{2}^{2}}-\tau_{y}(d)\right)}{\epsilon}\left(\frac{\tau_{1} D \tau_{1}+\tau_{2} D \tau_{2}}{\sqrt{\left(\tau_{1}^{2}\right)+\left(\tau_{2}\right)^{2}}}-\tau_{y}^{\prime}(d) D d\right) .
$$


In equations $(3.30)-(3.32)$

$$
H(x)=\left\{\begin{array}{ll}
0, & x<0 \\
1, & x \geq 0
\end{array} .\right.
$$

If we now multiply (3.27) by $D \tau_{1},(3.28)$ by $D \tau_{2},(3.29)$ by $D u,(3.32)$ by $\frac{\mu}{\mu+\beta} \tau_{y}^{\prime}(d) D d$ and add the resulting expressions we find that (3.1) and (3.2) hold where now

$$
\text { 4) } f=\frac{1}{2}\left(\frac{\mu\left(\left(D \tau_{1}\right)^{2}+\left(D \tau_{2}\right)^{2}\right)}{\mu+\beta}+\frac{\beta\left(\left(D\left(\tau_{1}+p_{1}\right)\right)^{2}+\left(D\left(\tau_{2}+p_{2}\right)\right)^{2}\right)}{\mu+\beta}+(D u)^{2}+\frac{\mu}{\mu+\beta} \tau_{y}^{\prime}(d)(D d)^{2}\right)
$$

$$
\begin{aligned}
& q_{1}=D u\left(\frac{\mu}{\mu+\beta} D \tau_{1}+\frac{\beta}{\mu+\beta} D\left(\tau_{1}+p_{1}\right)\right), \\
& q_{2}=D u\left(\frac{\mu}{\mu+\beta} D \tau_{2}+\frac{\beta}{\mu+\beta} D\left(\tau_{2}+p_{2}\right)\right),
\end{aligned}
$$

and

$$
\begin{aligned}
g & =\frac{\mu \tau_{y}^{\prime \prime}(d)}{2(\mu+\beta)} d_{t}(D d)^{2} \\
& -\frac{\mu}{\epsilon(\mu+\beta)} H\left(\sqrt{\tau_{1}^{2}+\tau_{2}^{2}}-\tau_{y}(d)\right)\left(\frac{\tau_{1} D \tau_{1}+\tau_{2} D \tau_{2}}{\sqrt{\tau_{1}^{2}+\tau_{2}^{2}}}-\tau_{y}^{\prime}(d) D d\right)^{2} \\
& -\frac{\mu}{\epsilon(\mu+\beta)} \frac{\left(\sqrt{\tau_{1}^{2}+\tau_{2}^{2}}-\tau_{y}(d)\right)_{+}}{\left(\tau_{1}^{2}+\tau_{2}^{2}\right)^{3 / 2}}\left(\tau_{2} D \tau_{1}-\tau_{1} D \tau_{2}\right)^{2} .
\end{aligned}
$$

The hypothesis that $\tau_{y}^{\prime \prime} \leq 0$ and $d_{t} \geq 0$ then guarantee that $g \leq 0$. Moreover, the boundary density satisfies

$$
q_{1} \cos \theta+q_{2} \sin \theta-f \leq-\frac{\mu\left(|D u|-\sqrt{\left(D \tau_{1}\right)^{2}+\left(D \tau_{2}\right)^{2}}\right)^{2}}{2(\mu+\beta)}
$$

$$
\frac{\beta\left(|D u|-\sqrt{\left(D\left(\tau_{1}+p_{1}\right)\right)^{2}+\left(D\left(\tau_{2}+p_{2}\right)\right)^{2}}\right)^{2}}{2(\mu+\beta)}-\frac{\tau_{y}^{\prime}(d)(D d)^{2}}{2} \leq 0
$$

and the latter two inequalities along with (3.2) yield the desired derivative bounds.

We conclude this section with an examination of the behavior of our system as the small parameter $\epsilon$ approaches zero from above. In what follows we let

$$
\Omega\left(x_{0}, y_{0}, R, t-s\right)=\left\{(x, y) \mid \sqrt{\left(x-x_{0}\right)^{2}+\left(y-y_{0}\right)^{2}} \leq R+t-s\right\}
$$

when $0 \leq s \leq t$ and

$$
B\left(x_{0}, y_{0}, R, t\right)=\left\{(x, y, s) \mid \sqrt{\left(x-x_{0}\right)^{2}+\left(y-y_{0}\right)^{2}} \leq R+t-s, 0 \leq s \leq t\right\}
$$


The a-priori estimates associated with (3.2) when $f$ is given by (3.3) or (3.34) and $D$ is one of the operators $\frac{\partial}{\partial t}, \frac{\partial}{\partial x}$, or $\frac{\partial}{\partial y}$ guarantee that if the initial values for a family $\left(\tau_{1}^{\epsilon}, \tau_{2}^{\epsilon}, p_{1}^{\epsilon}, p_{2}^{\epsilon}, d^{\epsilon}, u^{\epsilon}\right)$ of distributional solutions are in $L_{2}^{\text {loc }}$ independently of $\epsilon$, then we may choose a sequence $\epsilon_{i}, i=1,2, \ldots$, with the $\epsilon_{i}$ 's decreasing to zero and limit functions $\left(\tau_{1}^{0}, \tau_{2}^{0}, p_{1}^{0}, p_{2}^{0}, d^{0}, u^{0}\right)$ with the following properties:

(i) For any $\left(x_{0}, y_{0}\right), R>0$, and $t>0$ the sequence $\left(\tau_{1}^{\epsilon_{i}}, \tau_{2}^{\epsilon_{i}}, p_{1}^{\epsilon_{i}}, p_{2}^{\epsilon_{i}}, d^{\epsilon_{i}}, u^{\epsilon_{i}}\right)$ converge strongly in $L_{2}\left(B\left(x_{0}, y_{0}, R, t\right)\right)$ to $\left(\tau_{1}^{0}, \tau_{2}^{0}, p_{1}^{0}, p_{2}^{0}, d^{0}, u^{0}\right)$. Moreover, the limit functions have weak $t, x$, and $y$ derivatives and the sequences $D\left(\tau_{1}^{\epsilon_{i}} \tau_{2}^{\epsilon_{i}}, p_{1}^{\epsilon_{i}}, p_{2}^{\epsilon_{i}}, d^{\epsilon_{i}}, u^{\epsilon_{i}}\right)$ converge weakly in $L_{2}\left(B\left(x_{0}, y_{0}, R, t\right)\right)$ to $D\left(\tau_{1}^{0}, \tau_{2}^{0}, p_{1}^{0}, p_{2}^{0}, d^{0}, u^{0}\right)$ where again $D=\frac{\partial}{\partial t}, \frac{\partial}{\partial x}$ or $\frac{\partial}{\partial y}$.

(ii) The hypotheses (2.29) and (2.36) on the yield stress further guarantee that $\tau_{y}\left(d^{\epsilon_{i}}\right)$ converges strongly in $L_{2}\left(B\left(x_{0}, y_{0}, R, t\right)\right)$ to $\tau_{y}\left(d^{0}\right)$ and that $D \tau_{y}\left(d^{\epsilon_{i}}\right)=\tau_{y}^{\prime}\left(d^{\epsilon_{i}}\right) D d^{\epsilon_{i}}$ converges weakly in $L_{1}\left(B\left(x_{0}, y_{0}, R, t\right)\right)$ to $D \tau_{y}\left(d^{0}\right)=\tau_{y}^{\prime}\left(d^{0}\right) D d^{0}$ where once again $D=\frac{\partial}{\partial t}$, $\frac{\partial}{\partial x}$ or $\frac{\partial}{\partial y}$. Moreover, equations (2.31) -(2.42) and the convergence results (i) above imply that $\tau_{m}^{\epsilon_{i}} \frac{\partial p_{n}^{\epsilon_{i}}}{\partial t}, m$ and $n=1$ and 2 , and $\sqrt{\left(\tau_{1}^{\epsilon_{i}}\right)^{2}+\left(\tau_{2}^{\epsilon_{i}}\right)^{2}} \frac{\partial d^{\epsilon_{i}}}{\partial t}$ converge weakly in $L_{1}\left(B\left(x_{0}, y_{0}, R, t\right)\right)$ to $\tau_{m}^{0} \frac{\partial p_{n}^{0}}{\partial t}, m$ and $n=1$ and 2 , and $\sqrt{\left(\tau_{1}^{0}\right)^{2}+\left(\tau_{2}^{0}\right)^{2}} \frac{\partial d^{0}}{\partial t}$ respectively.

Finally, the identities

$$
\begin{aligned}
& \tau_{1}^{\epsilon_{i}} \frac{\partial p_{1}^{\epsilon_{i}}}{\partial t}+\tau_{2}^{\epsilon_{i}} \frac{\partial p_{2}^{\epsilon_{i}}}{\partial t}=\sqrt{\left(\tau_{1}^{\epsilon_{i}}\right)^{2}+\left(\tau_{2}^{\epsilon_{i}}\right)^{2}} \frac{\partial d^{\epsilon_{i}}}{\partial t} \\
& \tau_{2}^{\epsilon_{i}} \frac{\partial p_{1}^{\epsilon_{i}}}{\partial t}-\tau_{1}^{\epsilon_{i}} \frac{\partial p_{2}^{\epsilon_{i}}}{\partial t}=0
\end{aligned}
$$

guarantee that the limit functions satisfy

$$
\tau_{1}^{0} \frac{\partial p_{1}^{0}}{\partial t}+\tau_{2}^{0} \frac{\partial p_{1}^{0}}{\partial t}=\sqrt{\left(\tau_{1}^{0}\right)^{2}+\left(\tau_{2}^{0}\right)^{2}} \frac{\partial d^{0}}{\partial t}
$$

and

$$
\tau_{2}^{0} \frac{\partial p_{1}^{0}}{\partial t}-\tau_{1}^{0} \frac{\partial p_{2}^{0}}{\partial t}=0
$$

(iii) For any $\delta>0$, the measure

$$
m\left(\left\{(x, y, s) \in B\left(x_{0}, y_{0}, R, t\right) \mid \sqrt{\left(\tau_{1}^{0}\right)^{2}+\left(\tau_{2}^{0}\right)^{2}}-\tau_{y}\left(d^{0}\right) \geq \delta\right\}\right)=0 .
$$

The last identity implies that

$$
\begin{aligned}
& m\left(\left\{(x, y, s) \in B\left(x_{0}, y_{0}, R, t\right) \mid \sqrt{\left(\tau_{1}^{0}\right)^{2}+\left(\tau_{2}^{0}\right)^{2}}-\tau_{y}\left(d^{0}\right) \geq 0\right\}\right) \\
= & m\left(\left\{(x, y, s) \in B\left(x_{0}, y_{0}, R, t\right) \mid \sqrt{\left(\tau_{1}^{0}\right)^{2}+\left(\tau_{2}^{0}\right)^{2}}-\tau_{y}\left(d^{0}\right)=0\right\}\right) .
\end{aligned}
$$

In what follows we shall refer to

$$
\left\{(x, y, s) \in B\left(x_{0}, y_{0}, R, t\right) \mid \sqrt{\left(\tau_{1}^{0}\right)^{2}+\left(\tau_{2}^{0}\right)^{2}}-\tau_{y}\left(d^{0}\right)=0\right\}
$$


as the yield set. On open subsets $\mathcal{U}$ of the yield set we introduce $\Theta$ by

$$
\tau_{1}^{0}=\tau_{y}\left(d^{0}\right) \cos \Theta \text { and } \tau_{2}^{0}=\tau_{y}\left(d^{0}\right) \sin \Theta
$$

The relationships (3.41) and (3.42) then imply that

$$
\cos \Theta \frac{\partial p_{1}^{0}}{\partial t}+\sin \Theta \frac{\partial p_{2}^{0}}{\partial t}=\frac{\partial d^{0}}{\partial t}
$$

and

$$
\sin \Theta \frac{\partial p_{1}^{0}}{\partial t}-\cos \Theta \frac{\partial p_{2}^{0}}{\partial t}=0
$$

Since the weak limits $\left(\tau_{1}^{0}, \tau_{2}^{0}, p_{1}^{0}, p_{2}^{0}, u^{0}\right)$ also satisfy the conservation laws $(2.37)-(2.39)$ we find that in $\mathcal{U}$ the following equations are satisfied in the distributional sense

$$
\cos \Theta \tau_{y}^{\prime}\left(d^{0}\right) \frac{\partial d^{0}}{\partial t}-\tau_{y}\left(d^{0}\right) \sin \Theta \frac{\partial \Theta}{\partial t}-\frac{\partial u^{0}}{\partial x}=-\frac{\partial p_{1}^{0}}{\partial t}
$$

$$
\sin \Theta \tau_{y}^{\prime}\left(d^{0}\right) \frac{\partial d^{0}}{\partial t}+\tau_{y}\left(d^{0}\right) \cos \Theta \frac{\partial \Theta}{\partial t}-\frac{\partial u^{0}}{\partial y}=-\frac{\partial p_{2}^{0}}{\partial t}
$$

and

$$
\frac{\partial u^{0}}{\partial t}-\frac{\partial}{\partial x}\left(\tau_{y}\left(d^{0}\right) \cos \Theta+\frac{\beta}{\mu+\beta} p_{1}^{0}\right)-\frac{\partial}{\partial y}\left(\tau_{y}\left(d^{0}\right) \sin \Theta+\frac{\beta}{\mu+\beta} p_{2}^{0}\right)=0 .
$$

These equations represent a closed system for $\left(p_{1}^{0}, p_{2}^{0}, d^{0}, u^{0}, \Theta\right)$ on the yield surface. They imply that (3.52) holds and that

$$
\begin{gathered}
\left(1+\tau_{y}^{\prime}\left(d^{0}\right)\right) \frac{\partial d^{0}}{\partial t}=\cos \Theta \frac{\partial u^{0}}{\partial x}+\sin \Theta \frac{\partial u^{0}}{\partial y}, \\
\frac{\partial \Theta}{\partial t}=\frac{1}{\tau_{y}\left(d^{0}\right)}\left(-\sin \Theta \frac{\partial u^{0}}{\partial x}+\cos \Theta \frac{\partial u^{0}}{\partial y}\right) \\
\frac{\partial p_{1}^{0}}{\partial t}=\frac{\cos \Theta}{1+\tau_{y}^{\prime}\left(d^{0}\right)}\left(\cos \Theta \frac{\partial u^{0}}{\partial x}+\sin \Theta \frac{\partial u^{0}}{\partial y}\right)
\end{gathered}
$$

and

$$
\frac{\partial p_{2}^{0}}{\partial t}=\frac{\sin \Theta}{1+\tau_{y}^{\prime}\left(d^{0}\right)}\left(\cos \Theta \frac{\partial u^{0}}{\partial x}+\sin \Theta \frac{\partial u^{0}}{\partial y}\right)
$$

Not surprisingly, we find that in open sets $\mathcal{E}$ of $\left\{(x, y, s) \in B\left(x_{0}, y_{0}, R, t\right) \mid 0<\sqrt{\left(\tau_{1}^{0}\right)^{2}+\left(\tau_{2}^{0}\right)^{2}}<\tau_{y}\left(d^{0}\right)\right\}$ the weak limits satisfy the elasticity equations

$$
\begin{aligned}
& \frac{\partial \tau_{1}^{0}}{\partial t}-\frac{\partial u^{0}}{\partial x}=0 \\
& \frac{\partial \tau_{2}^{0}}{\partial t}-\frac{\partial u^{0}}{\partial y}=0 .
\end{aligned}
$$




$$
\frac{\partial u^{0}}{\partial t}-\frac{\partial \tau_{1}^{0}}{\partial x}-\frac{\partial \tau_{2}^{0}}{\partial y}=\frac{\beta}{\mu+\beta}\left(\frac{\partial p_{1}^{0}}{\partial x}+\frac{\partial p_{2}^{0}}{\partial y}\right)
$$

and

$$
\frac{\partial p_{1}^{0}}{\partial t}=\frac{\partial p_{2}^{0}}{\partial t}=\frac{\partial d^{0}}{\partial t}=0
$$

The assertions in part (i) which pertain to $\left(\tau_{1}^{\epsilon_{i}}, \tau_{2}^{\epsilon_{i}}, p_{1}^{\epsilon_{i}}, p_{2}^{\epsilon_{i}}, u^{\epsilon_{i}}\right)$ follow from (3.2), (3.3), and (3.34). Equations (2.29), (2.36), (3.2), and $\frac{\partial d^{\epsilon_{i}}}{\partial t} \geq 0$ imply that the $d^{\epsilon_{i}}$ 's are bounded in $L_{1}\left(B\left(x_{0}, y_{0}, R, t\right)\right)$. Their $L_{2}$ boundedness follows from the inequality

$$
\left(d^{\epsilon_{i}}(x y, s)\right)^{2} \leq 2(d(x, y, 0))^{2}+2 s \int_{0}^{s}\left(\frac{\partial d^{\epsilon_{i}}}{\partial \eta}\right)^{2}(x, y, \eta) d \eta
$$

which in turn implies that

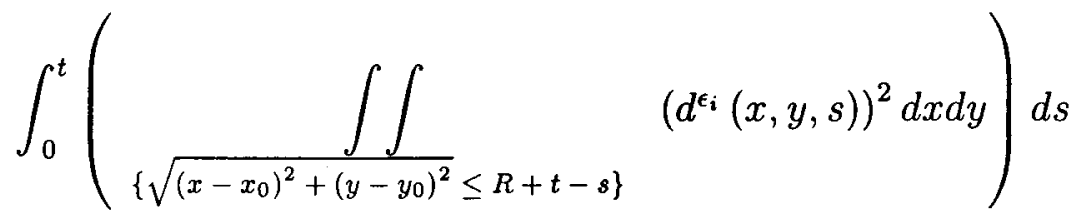

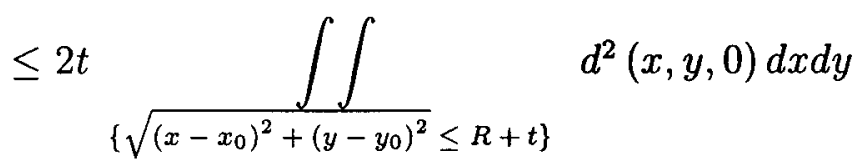

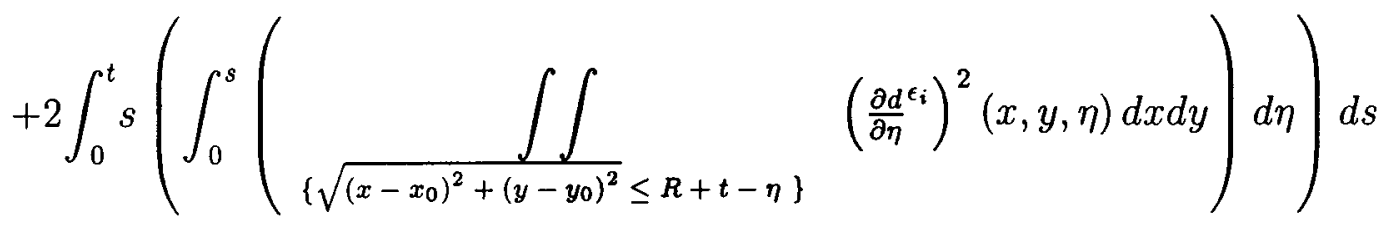

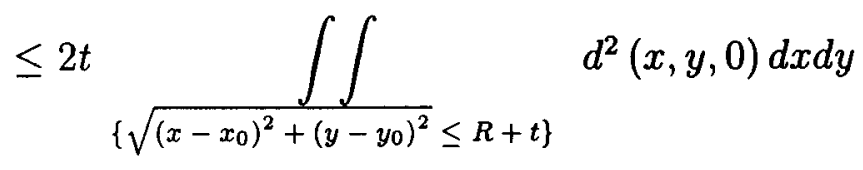

$$
\begin{aligned}
& +2 t^{2} \int_{0}^{t}\left(\int_{\left\{\sqrt{\left(x-x_{0}\right)^{2}+\left(y-y_{0}\right)^{2}} \leq R+t-\eta\right\}}\left(\frac{\partial d^{\epsilon_{i}}}{\partial \eta}\right)^{2}(x, y, \eta) d x d y\right) d \eta
\end{aligned}
$$

As noted previously, the assertions of (ii) follow directly from those of (i) and the governing equations $(2.40)-(2.42)$.

The veracity of (iii) follows from the inequality 


$$
\begin{aligned}
& 0 \leq \delta m\left\{(x, y, s) \in B\left(x_{0}, y_{0}, R, t\right) \mid \sqrt{\left(\tau_{1}^{0}\right)^{2}+\left(\tau_{2}^{0}\right)^{2}}-\tau_{y}\left(d^{o}\right) \geq \delta\right\} \\
& \leq \quad \iiint_{\left\{(x, y, s) \in B\left(x_{0}, y_{0}, R, t\right) \mid \sqrt{\left(\tau_{1}^{0}\right)^{2}+\left(\tau_{2}^{0}\right)^{2}}-\tau_{y}\left(d^{0}\right) \geq \delta\right\}}\left(\sqrt{\left(\tau_{1}^{0}\right)^{2}+\left(\tau_{2}^{0}\right)^{2}}-\tau_{y}\left(d^{0}\right)\right) d x d y d s \\
& =\underset{\left\{(x, y, s) \in B\left(x_{0}, y_{0}, R, t\right) \mid \sqrt{\left(\tau_{1}^{0}\right)^{2}+\left(\tau_{2}^{0}\right)^{2}}-\tau_{y}\left(d^{0}\right) \geq \delta\right\}}{ }\left(\sqrt{\left(\tau_{1}^{0}\right)^{2}+\left(\tau_{2}^{0}\right)^{2}}-\sqrt{\left(\tau_{1}^{\epsilon_{i}}\right)^{2}+\left(\tau_{2}^{\epsilon_{i}}\right)^{2}}\right) d x d y d s \\
& +\quad \iiint_{\left\{(x, y, s) \in B\left(x_{0}, y_{0}, R, t\right) \mid \sqrt{\left(\tau_{\mathbf{1}}^{0}\right)^{2}+\left(\tau_{2}^{0}\right)^{2}}-\tau_{y}\left(d^{0}\right) \geq \delta\right\}}\left(\tau_{y}\left(d^{\epsilon_{i}}\right)-\tau_{y}\left(d^{0}\right)\right) d x d y d s \\
& +\iiint_{\left\{(x, y, s) \in B\left(x_{0}, y_{0}, R, t\right) \mid \sqrt{\left(\tau_{1}^{0}\right)^{2}+\left(\tau_{2}^{0}\right)^{2}}-\tau_{y}\left(d^{0}\right) \geq \delta\right\}}\left(\sqrt{\left(\tau_{1}^{\epsilon_{i}}\right)^{2}+\left(\tau_{2}^{\epsilon_{i}}\right)^{2}}-\tau_{y}\left(d^{\epsilon_{i}}\right)\right) d x d y d s,
\end{aligned}
$$

the strong convergence results of part (i) which guarantee that the first two integrals on the right-hand side of (3.63) converge to zero as the $\epsilon_{i}$ 's tend to zero, and from the observation that the third integral is bounded from above by

$$
\iiint_{\mathrm{B}\left(x_{0}, y_{0}, R, t\right)}\left(\sqrt{\left(\tau_{1}^{\epsilon_{i}}\right)^{2}+\left(\tau_{2}^{\epsilon_{i}}\right)^{2}}-\tau_{y}\left(d^{\epsilon_{i}}\right)\right)_{+} d x d y d s
$$

which in turn is bounded by

$$
\left(m\left(B\left(x_{0}, y_{0}, R, t\right)\right)\right)^{1 / 2}\left(\iint_{B\left(x_{0}, y_{0}, R, t\right)}\left(\left(\sqrt{\left(\tau_{1}^{\epsilon_{i}}\right)^{2}+\left(\tau_{2}^{\epsilon_{i}}\right)^{2}}-\tau_{y}\left(d^{\epsilon_{i}}\right)\right)_{+}\right)^{2} d x d y d s\right)^{1 / 2} .
$$

The identity (3.2) with $f$ given by (3.3) and $g$ by (3.6) guarantees that

$$
\iiint_{B\left(x_{0}, y_{0}, R, t\right)}\left(\left(\sqrt{\left(\tau_{1}^{\epsilon_{i}}\right)^{2}+\left(\tau_{2}^{\epsilon_{i}}\right)^{2}}-\tau_{y}\left(d^{\epsilon_{i}}\right)\right)_{+}\right)^{2} d x d y d s
$$

$$
\leq \epsilon_{i} \frac{(\mu+\beta)}{\mu} \underset{\left\{\sqrt{\left(x-x_{0}\right)^{2}+\left(y-y_{0}\right)^{2}} \leq R+t\right\}}{\int}\left(\frac{\mu\left(\tau_{1}^{2}+\tau_{2}^{2}\right)}{2(\mu+\beta)}+\frac{\beta\left(\left(\tau_{1}+p_{1}\right)^{2}+\left(\tau_{2}+p_{2}\right)^{2}\right)^{2}}{2(\mu+\beta)}+\frac{u^{2}}{2}+\frac{\mu}{\mu+\beta} \int_{0}^{d} \tau_{y}(\eta) d \eta\right)(x, y, 0) d x d y
$$

and (3.64) and (3.65) imply that the third integral on the right-hand side of (3.63) tends to zero as $\epsilon_{i}$ tends to zero.

To establish (3.57)-(3.60) in open subsets $\mathcal{E}$ of $\left\{(x, y, s) \in B\left(x_{0}, y_{0}, R t\right) \mid 0<\sqrt{\left(\tau_{1}^{0}\right)^{2}+\left(\tau_{2}^{0}\right)^{2}}<\tau_{y}\left(d^{0}\right)\right\}$ it suffices to show that $\frac{\partial d^{0}}{\partial t}=0$ on $\mathcal{E}$. Equations (3.43) and (3.44) will then guarantee that $\frac{\partial p_{1}^{0}}{\partial t}=\frac{\partial p_{2}^{0}}{\partial t}=0$ and these identities, along with (2.37)-(2.39) will guarantee that (3.57)-(3.60) hold. 
In what follows we let $\delta>0$,

$$
E_{\delta}=\left\{(x, y, s) \in B\left(x_{0}, y_{0}, R, t\right) \mid \sqrt{\left(\tau_{1}^{0}\right)^{2}+\left(\tau_{2}^{0}\right)^{2}}-\tau_{y}\left(d^{0}\right) \leq-\delta<0\right\} .
$$

and observe that

$$
\begin{aligned}
\left|\iint_{E_{\delta}} \frac{\partial d^{0}}{\partial s} d x d y d s\right| & \leq\left|\iiint_{E_{\delta}}\left(\frac{\partial d^{0}}{\partial s}-\frac{\partial d^{\epsilon_{i}}}{\partial s}\right) d x d y d s\right| \\
+ & \left|\iiint_{E_{\delta} \cap\left\{\frac{\partial d^{\epsilon_{i}}}{\partial s}>0\right\}} \frac{\partial d^{\epsilon_{i}}}{\partial s} d x d y d s\right|
\end{aligned}
$$

The weak convergence of $\frac{\partial d^{\epsilon_{i}}}{\partial s}$ to $\frac{\partial d^{0}}{\partial s}$ guarantees that the first integral on the right-hand side of (3.67) may be made arbitrarily small. We estimate the second integral by

$$
\left(\iiint_{B\left(x_{0}, y_{0}, R, t\right)}\left(\frac{\partial d}{\partial s} \epsilon^{\epsilon_{i}}\right)^{2} d x d y d s\right)^{1 / 2} m\left(E_{\delta} \cap\left\{\frac{\partial d^{\epsilon_{i}}}{\partial s}>0\right\}\right)^{1 / 2} .
$$

That the first factor is bounded follows from (3.2) with $f$ given by (3.34) and $D=\frac{\partial}{\partial t}$. Thus, it suffices to show that $\lim _{i \rightarrow \infty} m\left(E_{\delta} \cap\left\{\frac{\partial d^{\epsilon_{i}}}{\partial s}>0\right\}\right)=0$. To establish this assertion we observe that $\left\{\frac{\partial d^{\epsilon_{i}}}{\partial s}>0\right\}=$ $\left\{\sqrt{\left(\tau_{1}^{\epsilon_{i}}\right)^{2}+\left(\tau_{2}^{\epsilon_{i}}\right)^{2}}-\tau_{y}\left(d^{\epsilon_{i}}\right)>0\right\}$, and that

$$
\begin{aligned}
& \delta m\left(E_{\delta} \cap\left\{\frac{\partial d^{\epsilon_{i}}}{\partial s}>0\right\}\right) \leq \underset{E_{\delta} \cap\left\{\frac{\partial d}{\partial s} \epsilon_{i}>0\right\}}{ }\left(\tau_{y}\left(d^{0}\right)-\sqrt{\left(\tau_{1}^{0}\right)^{2}+\left(\tau_{2}^{0}\right)^{2}}\right) d x d y d s \\
& \leq \quad \iiint_{E_{\delta} \cap\left\{\frac{\partial d}{\partial s} \epsilon_{i}>0\right\}}\left(\tau_{y}\left(d^{0}\right)-\tau_{y}\left(d^{\epsilon_{i}}\right)\right) d x d y d s \\
& +\quad \iiint_{E_{\delta} \cap\left\{\frac{\partial d}{\partial s} \epsilon_{i}>0\right\}}\left(\sqrt{\left(\tau_{1}^{\epsilon_{i}}\right)^{2}+\left(\tau_{2}^{\epsilon_{i}}\right)^{2}}-\sqrt{\left(\tau_{1}^{0}\right)^{2}+\left(\tau_{2}^{0}\right)^{2}}\right) d x d y d s .
\end{aligned}
$$

The strong convergence results of (i) imply that the latter two integrals tend to zero as the $\epsilon_{i}$ 's tend to zero thereby yielding $\lim _{i \rightarrow \infty} m\left(E_{\delta} \cap\left\{\frac{\partial d}{\partial s} \epsilon_{i}>0\right\}\right)=0$. 


\section{Computational Experiments}

In this section we present some computational experiments for the dimensionless system (2.37) - (2.42) when the normalized yield stress is given by

$$
\tau_{y}=1+c_{1}+\left(c_{1}-c_{2}\right) d-\frac{c_{1}}{1+d}
$$

and

$$
0<c_{2}<c_{1} .
$$

Since the flows associated with this system may be quite complicated we restrict our attention to problems with Riemann type data where

$$
\left(\tau_{1}, \tau_{2}, p_{1}, p_{2}, d\right)\left(x, y, 0^{+}\right) \equiv(0,0,0,0,0)
$$

and

$$
u\left(x, y, 0^{+}\right)=\left\{\begin{aligned}
u_{0}, & \text { if } \quad x y>0 \\
-u_{0}, & \text { if } \quad x y<0
\end{aligned}\right.
$$

where $u_{0}$ is a constant. The solutions generated by this data exhibit a high degree of symmetry and thus when visualizing them we may confine our attention to one of the four quadrants $(k-1) \frac{\pi}{2} \leq \theta \leq \frac{k \pi}{2}$, $k=1, \ldots, 4$. The data for $u\left(x, y, 0^{+}\right)$is not $H_{1}^{\text {loc }}$ but the functions

$$
u^{h}\left(x, y, 0^{+}\right)=\left\{\begin{array}{c}
u_{0}, \text { if } x>\frac{h}{2} \text { and } y>\frac{h}{2} \text { or } x<-\frac{h}{2} \text { and } y<-\frac{h}{2}, \\
-\quad u_{0}, \text { if } x<-\frac{h}{2} \text { and } y>\frac{h}{2} \text { or } x>\frac{h}{2} \text { and } y<-\frac{h}{2} \\
-\quad u_{0}+\frac{2 u_{0}}{h}\left(x+\frac{h}{2}\right), \text { if }-\frac{h}{2} \leq x \leq h / 2 \text { and } y \geq \frac{h}{2}, \\
u_{0}-\frac{2 u_{0}}{h}\left(x+\frac{h}{2}\right), \text { if }-\frac{h}{2} \leq x \leq \frac{h}{2} \text { and } y \leq-\frac{h}{2}, \\
u_{0}-\frac{2 u_{0}}{h}\left(y+\frac{h}{2}\right), \text { if }-\frac{h}{2} \leq y \leq \frac{h}{2} \text { and } x \leq-\frac{h}{2}, \\
-\quad u_{0}+\frac{2 u_{0}}{h}\left(y+\frac{h}{2}\right), \text { if }-\frac{h}{2} \leq y \leq \frac{h}{2} \text { and } x \geq \frac{h}{2} \\
u_{0}-\frac{2 u_{0}}{h}\left(x+\frac{h}{2}\right)-\frac{2 u_{0}}{h}\left(y+\frac{h}{2}\right)+\frac{3 u_{0}}{h^{2}}\left(x+\frac{h}{2}\right) \\
\text { if }-\frac{h}{2} \leq x \leq \frac{h}{2} \text { and }-\frac{h}{2} \leq y \leq \frac{h}{2}
\end{array}\right.
$$

are $H_{1}^{\text {loc }}$ and this, together with our $L_{2}^{\text {loc }}$ contractivity estimate of the previous section, is sufficient to guarantee that the solution to $(2.37)-(2.42)$ taking on the data (4.3) and (4.5) has a strong $L_{2}^{\text {loc }}$ limit as 
$h \rightarrow 0^{+}$which satisfies (2.37) - (2.42), (4.3), and (4.4). This limiting behavior is true when $\epsilon>0$ is fixed and also in the $\epsilon=0^{+}$limit when the rate independent equations (3.52) - (3.60) govern.

Our updating algorithm is as follows. We assume we are given $\left(\tau_{1}, \tau_{2}, p_{1}, p_{2}, d, u\right)^{N}(x, y)$ on the $x-y$ plane. These represent the approximate solution at time $t=(N-1 / 2) \delta$ where $\delta$ is our time step and $N \geq 1$. To advance these data we successively solve the following systems:

$$
\begin{aligned}
& \left\{\begin{array}{c}
\frac{\partial \tau_{1}}{\partial t}-\frac{\partial u}{\partial x}=0, \quad \frac{\partial \tau_{2}}{\partial t}=0, \quad \frac{\partial u}{\partial t}-\frac{\partial}{\partial x}\left(\tau_{1}+\frac{\beta}{\mu+\beta} p_{1}\right)=0 \\
\text { and } \quad \frac{\partial p_{1}}{\partial t}=\frac{\partial p_{2}}{\partial t}=\frac{\partial d}{\partial t}=0, \quad 0 \leq t \leq \delta
\end{array}\right. \\
& \left\{\begin{array}{l}
\frac{\partial \tau_{1}}{\partial t}=0, \quad \frac{\partial \tau_{2}}{\partial t}-\frac{\partial u}{\partial y}=0, \quad \frac{\partial u}{\partial t}-\frac{\partial}{\partial y}\left(\tau_{2}+\frac{\beta}{\mu+\beta} p_{2}\right)=0 \\
\text { and } \quad \frac{\partial p_{1}}{\partial t}=\frac{\partial p_{2}}{\partial t}=\frac{\partial d}{\partial t}=0, \quad 0 \leq t \leq \delta
\end{array}\right.
\end{aligned}
$$

and

$$
\begin{cases}\frac{\partial}{\partial t}\left(\tau_{1}+p_{1}\right)=\frac{\partial}{\partial t}\left(\tau_{2}+p_{2}\right)=\frac{\partial u}{\partial t}=0, & \\ \frac{\partial p_{1}}{\partial t}=\frac{\tau_{1}\left(\sqrt{\tau_{1}^{2}+\tau_{2}^{2}}-\tau_{y}(d)\right)_{+}}{\epsilon \sqrt{\tau_{1}^{2}+\tau_{2}^{2}}}, \quad \frac{\partial p_{2}}{\partial t}=\frac{\tau_{2}\left(\sqrt{\tau_{1}^{2}+\tau_{2}^{2}}-\tau_{y}(d)\right)_{+}}{\epsilon \sqrt{\tau_{1}^{2}+\tau_{2}^{2}}} \\ \text { and } \frac{\partial d}{\partial t}=\frac{\left(\sqrt{\tau_{1}^{2}+\tau_{2}^{2}}-\tau_{y}(d)\right)_{+}}{\epsilon}, \quad 0 \leq t \leq \delta .\end{cases}
$$

Our principal reason for this splitting is that the systems (4.6) and (4.7) may be updated exactly by elementary characteristic methods and (4.8) may be easily integrated to any desired order of accuracy via Runge Kutta methods.

For (4.6) we use $\left(\tau_{1}, \tau_{2}, p_{1}, p_{2}, d, u\right)^{N}$ as initial data and let $\left(\tau_{1}^{1}, \tau_{2}^{1}, p_{1}^{1}: p_{2}^{1}, d^{1}, u^{1}\right)$ denote the solution to (4.6) with these data at time $t=\delta$. We then solve (4.7) using $\left(\tau_{1}^{1}, \tau_{2}^{1}, p_{1}^{1}, p_{2}^{1}, d, u^{1}\right)$ as initial data and let $\left(\tau_{1}^{2}, \tau_{2}^{2}, p_{1}^{2}, p_{2}^{2}, d^{2}, u^{2}\right)$ denote the solution at $t=\delta$. We next repeat the process but first solve (4.7) with the data $\left(\tau_{1}, \tau_{2}, p_{1}, p_{2}, d, u\right)^{N}$ and let $\left(\tau_{1}^{3}, \tau_{2}^{3}, p_{1}^{3}, p_{2}^{3}, d^{3}, u^{3}\right)$ denote the solution at $t=\delta$. We then use $\left(\tau_{1}^{3}, \tau_{2}^{3}, p_{1}^{3}, p_{2}^{3}, d^{3}, u^{3}\right)$ as data for (4.6) and let $\left(\tau_{1}^{4}, \tau_{2}^{4}, p_{1}^{4}, p_{2}^{4}, d^{4}, u^{4}\right)$ denote the solution at $t=\delta$. Finally we average the approximate solutions indexed by (2) and (4) and denote the result as $\left(\tau_{1}^{5}, \tau_{2}^{5}, p_{1}^{5}, p_{2}^{5}, d^{5}, u^{5}\right)$; that is

$$
\left(\tau_{1}^{5}, \tau_{2}^{5}, p_{1}^{5}, p_{2}^{5}, d^{5}, u^{5}\right)=\frac{1}{2}\left(\tau_{1}^{2}+\tau_{1}^{4}, \tau_{2}^{2}+\tau_{2}^{4}, p_{1}^{2}+p_{1}^{4}, p_{2}^{2}+p_{2}^{4}, d^{2}+d^{4}, u^{2}+u^{4}\right)
$$

We note that this particular approximation represents a second order update to the "elastic" wave equation:

$$
\left\{\begin{array}{l}
\frac{\partial \tau_{1}}{\partial t}-\frac{\partial u}{\partial x}=0, \quad \frac{\partial \tau_{2}}{\partial t}-\frac{\partial u}{\partial y}=0, \quad \frac{\partial u}{\partial t}-\frac{\partial}{\partial x}\left(\tau_{1}+\frac{\beta p_{1}}{\mu+\beta}\right)-\frac{\partial}{\partial y}\left(\tau_{2}+\frac{\beta p_{2}}{\mu+\beta}\right)=0 \\
\frac{\partial p_{1}}{\partial t}=\frac{\partial p_{2}}{\partial t}=\frac{\partial d}{\partial t}=0, \quad 0 \leq t \leq \delta
\end{array}\right.
$$


taking on the data $\left(\tau_{1}, \tau_{2}, p_{1}, p_{2}, d, u\right)^{N}$ at $t=0$ and does better than either of the approximates labeled 2 or 4; in particular solution symmetries are preserved via the averaging algorithm.

The final step in our algorithm involves solving (4.8) with the data $\left(\tau_{1}^{5}, \tau_{2}^{5}, p_{1}^{5}, p_{2}^{5}, d^{5}, u^{5}\right)$. Over the interval $0 \leq t \leq \delta$ we have

$$
\tau_{1}+p_{1} \equiv \tau_{1}^{5}+p_{1}^{5}, \tau_{2}+p_{2} \equiv \tau_{2}^{5}+p_{2}^{5}, \text { and } u \equiv u^{5}
$$

and

$$
\left\{\begin{array}{l}
\frac{\partial \tau_{1}}{\partial t}=\frac{\tau_{1}\left(\sqrt{\tau_{1}^{2}+\tau_{2}^{2}}-\tau_{y}(d)\right)_{+}}{\epsilon \sqrt{\tau_{1}^{2}+\tau_{2}^{2}}}, \quad \frac{\partial \tau_{2}}{\partial t}=\frac{\tau_{2}\left(\sqrt{\tau_{1}^{2}+\tau_{2}^{2}}-\tau_{y}(d)\right)_{+}}{\epsilon \sqrt{\tau_{1}^{2}+\tau_{2}^{2}}} \\
\text { and } \frac{\partial d}{\partial t}=\frac{\left(\sqrt{\tau_{1}^{2}+\tau_{2}^{2}}-\tau_{y}(d)\right)_{+}}{\epsilon} .
\end{array}\right.
$$

If we let

$$
\tau_{1}=J \cos \Theta \text { and } \tau_{2}=J \sin \Theta
$$

then equation (4.12) implies

$$
J+d \equiv J^{5}+d^{5} \quad \text { and } \quad \Theta \equiv \Theta^{5}, \quad 0 \leq t \leq \delta
$$

and

$$
\frac{\partial d}{\partial t}=\frac{\left(J^{5}+d^{5}-d-\tau_{y}(d)\right)_{+}}{\epsilon}, \quad 0 \leq t \leq \delta
$$

In (4.14), $J^{5}=\sqrt{\left(\tau_{1}^{5}\right)^{2}+\left(\tau_{2}^{5}\right)^{2}}$ and $0 \leq \Theta^{5}<2 \pi$ satisfies

$$
\cos \Theta^{5}=\frac{\tau_{1}^{5}}{J^{5}} \text { and } \sin \Theta^{5}=\frac{\tau_{2}^{5}}{J^{5}} .
$$

In what follows we let $d^{6}$ denote our update of (4.15) taking on the data $d^{5}$ at $t=0$. Equation (4.14) then implies that

$$
\left\{\begin{array}{l}
J^{6}=J^{5}+d^{5}-d^{6}, \quad \tau_{1}^{6}=J^{6} \frac{\tau_{1}^{5}}{J^{5}}, \quad \tau_{2}^{6}=J^{6} \frac{\tau_{2}^{5}}{J^{5}} \\
u^{6}=u^{5}, \quad p_{1}^{6}=p_{1}^{5}+\tau_{1}^{5}-\tau_{1}^{6}, \quad \text { and } \quad p_{2}^{6}=p_{2}^{5}+\tau_{2}^{5}-\tau_{2}^{6} .
\end{array}\right.
$$

Our approximate solution at $t=(N+1 / 2) \delta$ is given by the update labeled 6 . To obtain the approximate solution at $t=\delta / 2$ we merely solve (4.8) over the interval $0 \leq t \leq \delta / 2$ with the prescribed initial data and take the value of their update at $t=\delta / 2$ to be $\left(\tau_{1}, \tau_{2}, p_{1}, p_{2}, d, u\right)^{1}$.

The snapshots shown in Figure 1-18 were run with the normalized yield stress given by (4.1) when $c_{1}=1$ and $c_{2}=.5$. The parameter $u_{0}$ defining the initial data was set to 1.5 and we chose $\delta=h=.01$. The parameter $\epsilon$ was set to 0.1 . Surface renderings of $J=\sqrt{\tau_{1}^{2}+\tau_{2}^{2}}, d$, and $u$ are shown at times .3,.4, and .5 .

The purely one dimensional nature of the solutions away from the corner where strong interactions take place is evident from these simulations and it is clear from these calculations that our algorithm captures the sharp contact discontinuities in $J$ and $u$ correctly. Our algorithm is easy to implement and avoids a number of thorny issues we would have to contend with if we tried to integrate the reduced $\epsilon=0^{+}$ equations directly. 


\section{References}

[1] J. M. GREENBERG, Models of elastic-perfectly plastic materials, Euro. J. Appl. Math., 1(1990), pp. 131-150.

[2] J. M. GREENBERG, The longtime behavior of elastic-perfectly plastic materials, in Free Boundary Problems Involving Solids-Proceedings of the International Colloquium Free Boundary Problems: Theory and Applications, J. M. Chadam and H. K. Rasmussen, eds., Longman Scientific and Technical, Harlow, UK, 1993, pp. 28-34.

[3] J. M. GREENBERG AND ANNE NOURI Antiplane Shearing Motions of a Visco-plastic Solid, Siam J. Math. Anal., Vol. 24, No. 4, 1993, pp. 943-967.

[4] A. NOURI AND M. RASCLE, A Global Existence and Uniqueness Theorem for a Model Problem in Dynamic Elasto-Plasticity with Isotropic Strain-Hardening, Siam J. Math. Anal., Vol. 26, No. 4, 1995, pp. $850-868$

[5] M. MIHĂIlESCU-SUliCIU, I. SULICIU, AND W. O. WILliAMS, On Visco-plastic and ElasticPlastic Oscillators, Q. Applied Math., Vol. 47, No. 1, 1989, pp. 105-116. 